SLAC-PUB-8659

October 2000

astro-ph/0003270

\title{
The Origin Of Cosmic Rays: What Can GLAST Say?
}

\author{
Jonathan F. Ormes, Seth Digel, Igor V. Moskalenko, and Alexander Moiseev \\ Goddard Space Flight Center, Greenbelt, Maryland \\ Roger Williamson \\ HEPL, Stanford University, Stanford, CA. 94305 \\ on behalf of the GLAST collaboration
}

Presented at ACE 2000: The Acceleration and Transport of Energetic Particles Observed in the Heliosphere, 1/5/2000 - 1/8/2000, Indian Wells, CA, USA

Stanford Linear Accelerator Center, Stanford University, Stanford, CA 94309

Work supported by Department of Energy contract DE-AC03-76SF00515. 


\title{
The Origin of Cosmic Rays: What Can GLAST Say?
}

\author{
Jonathan F. Ormes, Seth Digel, Igor V. Moskalenko ${ }^{1}$, and Alexander Moiseev, \\ Goddard Space Flight Center, Greenbelt, Maryland \\ and Roger Williamson \\ Stanford University \\ on behalf of the GLAST collaboration
}

\begin{abstract}
Gamma rays in the band from $30 \mathrm{MeV}$ to $300 \mathrm{GeV}$, used in combination with direct measurements and with data from radio and X-ray bands, provide a powerful tool for studying the origin of Galactic cosmic rays. Gamma-ray Large Area Space Telescope (GLAST) with its fine 10-20 arcmin angular resolution will be able to map the sites of acceleration of cosmic rays and their interactions with interstellar matter. It will provide information that is necessary to study the acceleration of energetic particles in supernova shocks, their transport in the interstellar medium and penetration into molecular clouds.
\end{abstract}

\section{INTRODUCTION}

The identification of the sites of cosmic-ray acceleration is one of the main unsolved problems in Galactic cosmic-ray astrophysics. We know from radio [1] and recent $\mathrm{X}$-ray observations [2] of synchrotron radiation from supernova remnants that electrons are accelerated to $\mathrm{TeV}$ energies. Direct evidence for the acceleration of protons is not yet in hand. There is a considerable theoretical literature that quantifies how this acceleration takes place in the turbulent magnetic fields associated with the shock waves generated by supernova explosions propagating into the interstellar medium [3]. With the Gamma-ray Large Area Space Telescope (GLAST), proposed for launch in 2005 , we have the possibility of detecting $\gamma$ rays from the freshly accelerated cosmic-ray nuclei at their acceleration site. The optimal candidate sites will be those where the shock waves collide with either swept up interstellar matter or nearby clouds. The most commonly discussed site is individual supernova remnants [4] however multiple supernovas inside superbubbles have also been considered [5].

'NRC Resident Research Associate visiting GSFC from Inst. for Nucl. Phys., Moscow State U.

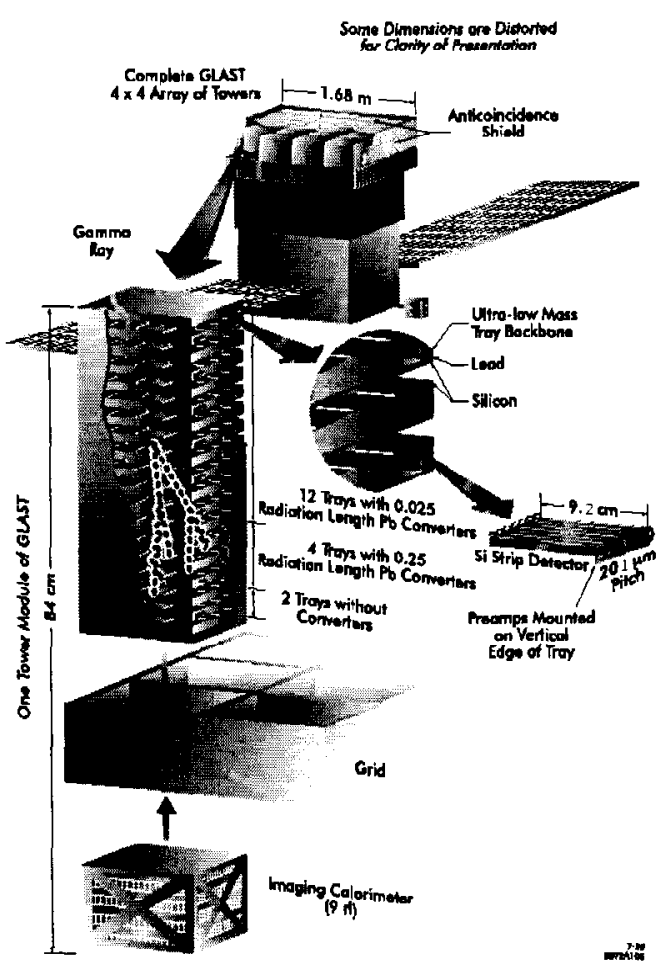

Fig. 1: The GLAST large area telescope 
While the great majority of the cosmic rays are nuclei, not electrons, most of the electromagnetic signatures of cosmic rays reflect electrons as seen in synchrotron radiation or inverse Compton (IC) interactions of photons with electrons. This includes even the highest energy band, photons around $1 \mathrm{TeV}$. To identify the site where the bulk of the energy is pumped into relativistic particles we need to find a signature of accelerated nucleons. Such a signature will be the spectrum reflective of neutral-pion decay from collisions of freshly-accclerated nuclei with nearby gas and dust ${ }^{2}$. Depending of the conditions in the medium the neutral-pion decay $\gamma$ rays will be evident as a spectral feature in the range $50 \mathrm{MeV}$ to a few $\mathrm{GeV}$, while above this range their spectral power law index will match the spectral index of the cosmic rays at the site. This range is ideal for studies with GLAST.

\section{GLAST CAPABILIES \& SNR}

The GLAST telescope we have proposed is shown in Figure 1. Its capabilities are summarized in Figure 2. At $1 \mathrm{GeV}$, the effective area, including inefficiencies due to photon conversion and background rejection, will be more than $1 \mathrm{~m}^{2}$, and our single photon angular resolution will be 0.4 degrees. Supernova remnants can be observed well off axis (up to 60 degrees) without loss of angular resolution and the $2.4 \mathrm{sr}$ solid angle allows any individual source to be observed at a $20 \%$ duty cycle. Thus, supernova remnants can be observed in the normal sky-scanning mode of GLAST operation. Minimal tails in the point-spread function (the $95 \%$ containment angle is typically 2.5 times the $68 \%$ containment angle) optimize the capability for mapping the structure of $\gamma$-ray emission. The challenge will be to find sources with angular scales large enough to be mapped with angular resolution sufficient to separate the extended shell emission from that of the central compact source, nominally a pulsar.

\begin{tabular}{|c|c|c|c|}
\hline \multicolumn{4}{|c|}{ TABLE 1. CANDIDATE SUPERNOVA } \\
\hline Remnant & $\begin{array}{c}\text { Distance } \\
\text { (kpc) }\end{array}$ & $\begin{array}{c}\text { Age } \\
\text { (years) }\end{array}$ & $\begin{array}{c}\text { Size } \\
\text { (arcmin) }\end{array}$ \\
\hline RXJ1713.7-3946 & 6 & $? ?$ & 70 \\
\hline$\gamma$ Cygni & 1.8 & 7000 & 60 \\
\hline IC443 & 1.5 & 5000 & 45 \\
\hline SN1006 & $\sim 3$ & 1000 & 30 \\
\hline CasA & 2.8 & 300 & 5 \\
\hline Kepler & 4.4 & 400 & 3 \\
\hline
\end{tabular}

\footnotetext{
${ }^{2} \mathrm{P}+\mathrm{P}->\pi^{\circ}+\mathrm{X}$ and $\pi^{\circ} \Rightarrow 2 \gamma$
}

The obvious candidates for this search are those SNR for which EGRET reported a finite flux [6]. We also include RXJ1713.7-3946 for which TeV emission has recently been reported [7]. They are listed in Table 1 along with relevant parameters. To predict the signal GLAST might detect for a typical case, we have modeled the $\gamma$ rays from $\gamma$ Cygni taking the total flux as measured by EGRET and dividing it into two separate components with $60 \%$ of the flux from the pulsar [8].
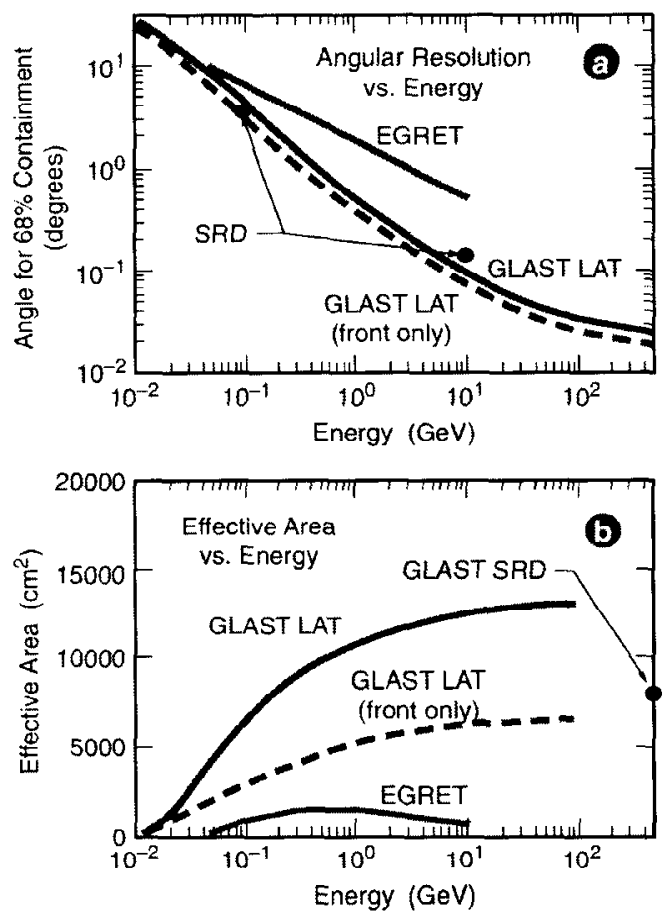

Fig. 2: Performance characteristics of GLAST

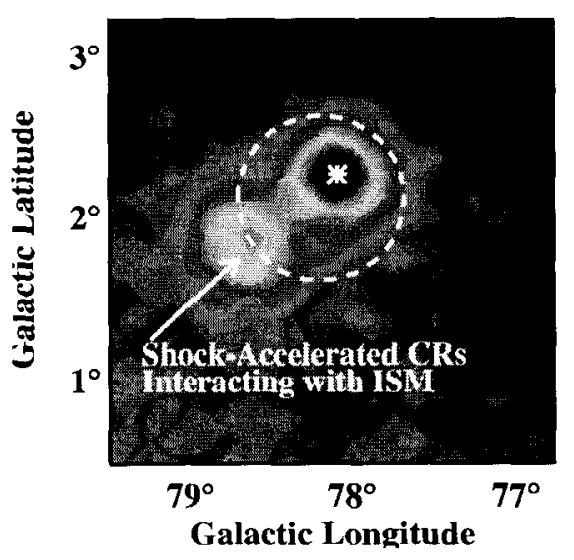

Fig. 3: Simulated observation of $\gamma$ Cygni 
The result of this simulation is shown in Figure 3. Note that the shell component is clearly resolved from the central point source. The spectrum of the $\gamma$ Cygni region is taken from Ref. 6 .

Assuming this source ( $\gamma$ Cygni) is as modeled we expect to spatially resolve the shell source and to be able to measure the spectrum [9] shown in Figure 4. The locally observed cosmic-ray nuclei have spectra proportional to $\mathrm{E}^{-2.7}$. This spectrum is the natural result of shock models which predict harder source spectra, $\mathrm{E}^{-\delta}$ with spectral exponents $\delta$ in the range 2.0 -2.3 combined with the energy dependent losses by diffusion. The IC and bremsstrahlung components can be deconvolved and subtracted to detcrmine the $\pi^{0}$ contribution. The asymptotic spectral exponent at encrgies above $10 \mathrm{GcV}$ should be indicative of the source spectrum of freshly-accelerated cosmic rays.

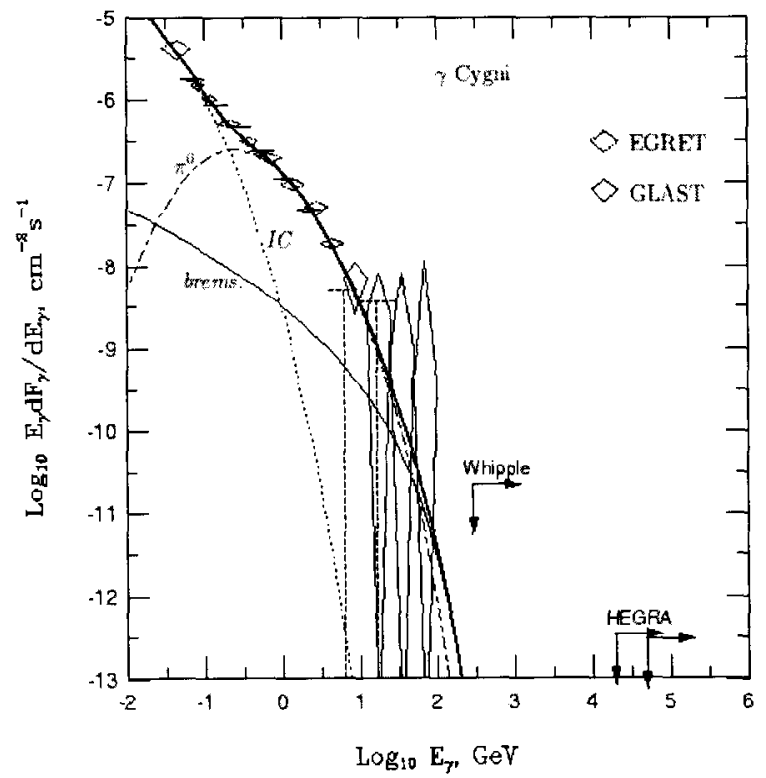

Fig. 4: Expected data from GLAST compared to one of the models of Gaisser et al. [9].

\section{DIFFUSE CONTINUUM GAMMA- RAYS}

Once accelerated, the cosmic rays quickly diffuse away from their sources, mix with those from other sources and diffuse out of the Galaxy. Most theoretical treatments of cosmic-ray transport from their sources to the Earth assume the result of this process is a steady flux of cosmic rays throughout the Galaxy with sources in equilibrium with losses.
With GLAST we have a chance to probe the spatial structure on a scale that can begin to test this hypothesis.

Of the many possible observables of cosmic-ray transport through the interstellar medium to earth (e.g. Be, B isotopes, positrons, antiprotons) the least exploited to date has been $\gamma$ rays. The diffuse $\gamma$-ray emission in any direction is a measure of the point by point product of the cosmic-ray intensity and the matter density in the interstellar medium. The secondary antiprotons and positrons are produced in the same collisional processes. The EGRET team has modeled this diffuse $\gamma$-ray emission [10] on 0.5 degree scale. Their models distribute the cosmic rays proportionally to the matter in the Galaxy on scales of order $1 \mathrm{kpc}$.

One of the most puzzling findings from EGRET is the excess in the Galactic diffuse $\gamma$-ray emission above $1 \mathrm{GeV}$ [11]. Independent confirmation and extension toward the higher energies would help to understand this excess. Is the spectrum of cosmicray nuclei [12] or electrons [13] elsewhere in the Galaxy different from what we measure locally? If so, what are the corresponding consequences for all cosmic-ray physics? The range of such possibilities have been recently discussed in the literature [14].

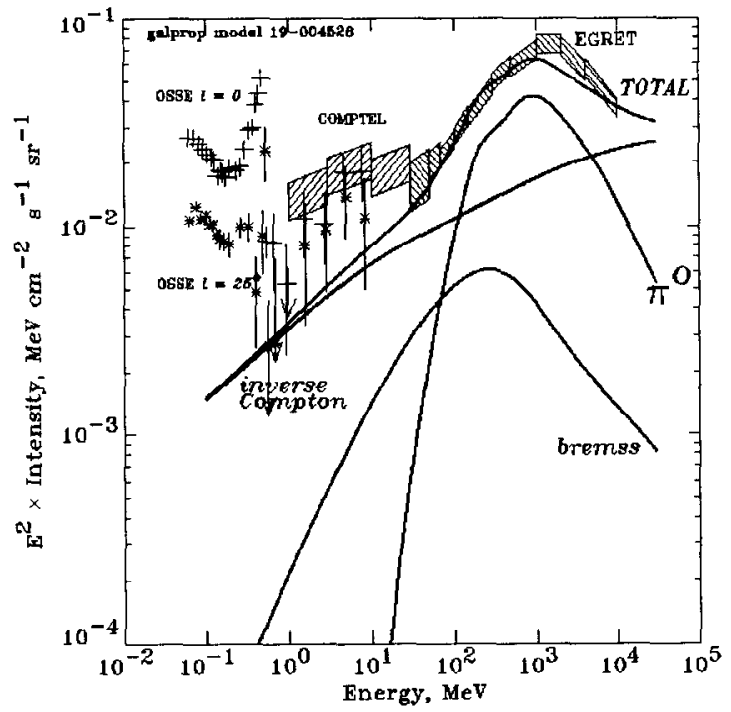

Fig. 5: A model [14] of the Galactic diffuse emission that fits the high energy EGRET data.

The self-consistent approach to this problem developed by Strong et al. [14] models the diffusive propagation of nucleons and electrons. Figure 5 shows their predictions of the various contributing components. Their conclusion, reflected in the 
figure, is that the harder electron spectrum in the Galaxy is more likely. But their solution is not unique and requires confirmation with the additional data that GLAST will provide. Measurements of high-energy antiproton and positron spectra provide a useful constraint on the nucleon spectrum, while the electron spectrum can be different from place to place due to the large electron energy losses (though radio measurements of synchrotron emission provide a constraint for some electron energies). These hypotheses can be tested with GLAST by measuring the spectrum of Galactic diffuse $\gamma$-ray emission above few GeV. First, one can distinguish betwecn the inverse Compton and neutral-pion decay components, and, second, the latitude and longitude $\gamma$ -ray profiles are different for gas-related nucleon and broader electron components.

Another result of the studies [15] is that the inverse Compton (IC) scattering, especially at high latitudes, plays a more important role than previously thought. The anisotropic distribution of photons above the Galactic plane will result in greater (up to $40 \%$ ) flux from IC in the halo. Evidence for a large $\gamma$ -ray halo has been found in an analysis of the EGRET data [16]. This affects our estimates of the extragalactic component, the intensity of cosmic rays in the Galaxy at large and the size of the Galactic cosmic-ray containment halo. The importance of high latitude IC can be tested with GLAST by measuring $\gamma$ ray profiles at high energies. Therefore, a major objective of GLAST will be to resolve the high latitude diffuse emission [17] and find, or at least greatly reduce, the fraction of that flux which is due to unresolved point sources.

A longstanding question in cosmic-ray astrophysics has been the extent to which cosmic rays can penetrate into clouds and interact with material there. The issue is one of the length scale of equipartition of energy between cosmic rays, thermal motion of interstellar gas and energy in magnetic fields. Equipartition seems to hold on the kpc scale, but what about scales below $1 \mathrm{kpc}$ ? GLAST will be able to study the $\gamma$-ray emission from molecular clouds, super-bubble walls and other interstellar concentrations of matter on an angular scale heretofore impossible reaching the tens of arc minute range. At a distance of $1 \mathrm{kpc}$, spatial scales of 3-10 parsec will be resolved.

\section{CONCLUSION}

Gamma rays are a powerful tool to explore the sources and distribution of cosmic rays in our Galaxy. In turn, having improved understanding of the role of cosmic rays is essential for the study of many topics in $\gamma$-ray astronomy to be addressed by GLAST. The results of the studies discussed here will have important consequences for understanding of the distribution of cosmic rays in the Galaxy and their dynamic effects thereupon. Besides, it is worth noting that complete understanding of both the Galactic cosmic rays and the $\gamma$-ray emissions are essential for the study of the dark matter in the Galaxy.

\section{REFERENCES}

1. Reynolds \& Gilmorc, 1993, Astronomical J., 106, 272

2. Koyama et al. 1995, Nature 378, 255; Allen et al. 1997, ApJ, 487, L97; Koyama et al. 1997 PASJ, 49, L7; Keohane et al. 1997 ApJ, 484, 356; Keohane PhD thesis; Tanimori et al. 1998, Ap.J, 497, L25

3. Baring et al. 1999, ApJ, 513, 311

4. Blandford, \& Ostriker, 1980, ApJ, 237, 793; Axford, Ann. NY Acad. Sci., 375, Acceleration of Cosmic Rays by Shock Waves, ed. Ramaty \& Jones, 297

5. Higdon, Lingenfelter \& Ramaty, 1998, ApJ 509, L33

6. Esposito et al. 1996, ApJ, 461, 820

7. Muraishi et al. 2000, A\&A, 354, L57

8. Brazier et al. 1996, MNRAS, 281, 1033

9. Gaisser, Protheroe \& Stanev, 1998, ApJ, 492, 219

10. Hunter et al. 1997, ApJ, 481, 205

11. Hunter et al. 1997, ApJ, 467, L33

12. Mori, 1997, ApJ 478, 225

13. Porter \& Protheroe, 1997, J. Phys. G: Nucl. Part. Phys. 23, 1765; Pohl \& Esposito 1998, ApJ, 507, 327

14. Moskalenko, Strong \& Reimer, 1998, 338, L75; Strong, Moskalenko \& Reimer, $2000 \mathrm{ApJ} 537$, in press (astro-ph/9811296); Moskalenko and Strong, 1998, ApJ, 493, 694

15. Moskalenko \& Strong, 2000, ApJ, 528, 357

16. Dixon et al. 1998, New Astron. 3(7), 539

17. Sreekumar et al., ApJ, 494, 52 


\title{
What Can GLAST Say About the Origin of Cosmic Rays in Other Galaxies
}

\author{
Seth Digel $^{\mathrm{a}, \mathrm{b}}$, Igor V. Moskalenko ${ }^{\mathrm{a}, \mathrm{c}}$, and Jonathan F. Ormes, \\ P. Sreekumar ${ }^{d,}$ and P. Roger Williamson ${ }^{\mathrm{e}}$, \\ ${ }^{2}$ NASA/Goddard Space Flight Center, Greenbelt, Maryland \\ 'Universities Space Research Association, \\ 'National Research Council and Institute for Nuclear Physics, \\ Moscow State University \\ ${ }^{\mathrm{d}}$ Indian Space Research Organization, ${ }^{\mathrm{e}}$ Stanford University \\ on behalf of the GLAST collaboration
}

Presented at ACE 2000: The Acceleration and Transport of Energetic Particles Observed in the Heliosphere, 1/5/2000-1/8/2000, Indian Wells, CA, USA 


\title{
What Can GLAST Say About the Origin of Cosmic Rays in Other Galaxies?
}

\author{
Seth W. Digel ${ }^{\mathrm{a}, \mathrm{b}}$, Igor V. Moskalenko ${ }^{\mathrm{a}, \mathrm{c}}$, Jonathan F. Ormes ${ }^{\mathrm{a}}$, \\ P. Sreekumard ${ }^{\mathrm{d}}$, and P. Roger Williamson ${ }^{\mathrm{e}}$, \\ on behalf of the GLAST collaboration \\ ${ }^{a}$ NASA/Goddard Space Flight Center, Greenbelt, Maryland, ${ }^{b}$ Universities Space Research Association, \\ 'National Research Council and Institute for Nuclear Physics, Moscow State University, \\ ${ }^{d}$ Indian Space Research Organization, "Stanford University
}

\begin{abstract}
Gamma rays in the band from $20 \mathrm{MeV}$ to $300 \mathrm{GeV}$, used in combination with data from radio and Xray bands, provide a powerful tool for studying the origin of cosmic rays in our sister galaxies Andromeda and the Magellanic Clouds. Gamma-ray Large Area Space Telescope (GLAST) will spatially resolve these galaxies and measure the spectrum and intensity of diffuse gamma radiation from the collisions of cosmic rays with gas and dust in them. Observations of Andromeda will give an external perspective on a spiral galaxy like the Milky Way. Observations of the Magellanic Clouds will permit a study of cosmic rays in dwarf irregular galaxies, where the confinement is certainly different and the massive star formation rate is much greater.
\end{abstract}

\section{INTRODUCTION}

High-energy gamma rays are produced in interactions of high-energy cosmic rays with interstellar matter and photons. From the resulting diffuse emission of gamma rays, the properties of the cosmic rays can be inferred (e.g., (5)). Gamma rays have proven to be a useful probe of cosmic rays in the Milky Way, but gamma-ray telescopes to date have lacked the sensitivity and angular resolution to permit the same kind of detailed study of cosmic rays in external galaxics.

The Gamma-ray Large Area Space Telescope (GLAST) is the next generation high-energy (20 MeV$300 \mathrm{GeV}$ ) gamma-ray astronomy mission. It is part of the strategic plan of NASA's Office of Space Science and is currently planned for launch in 2005. GLAST will have a factor of 30 greater sensitivity than the Energetic Gamma-Ray Experiment Telescope (EGRET), launched in 1991 on the Compton Gamma-Ray Observatory. Derived performance parameters for our proposed design for the GLAST instrument, which was selected by NASA in February 2000, are presented in Table 1 and Figure 1. See the companion paper by Ormes et al. for information about the design and instrumental response of GLAST, and the Web site http://glast.gsfc.nasa.gov for information about the mission.
Table 1. Selected Parameters for GLAST and EGRET

\begin{tabular}{|c|c|c|}
\hline & EGRET & GLAST \\
\hline Energy Range & $0.02-30 \mathrm{GeV}$ & $0.02-300 \mathrm{GeV}$ \\
\hline Field of View & $0.5 \mathrm{sr}$ & $2.4 \mathrm{sr}$ \\
\hline Peak Eff. Area & $1500 \mathrm{~cm}^{2}$ & $13,000 \mathrm{~cm}^{2}$ \\
\hline \multicolumn{3}{|l|}{ Point Source } \\
\hline Sensitivity* & 5 & 0.16 \\
\hline Source Location ${ }^{\dagger}$ & $5^{\prime}-90^{\prime}$ & $0.2^{\prime}-1^{\prime}$ \\
\hline Mission Life & & $\begin{array}{l}5 \text { years } \\
\text { (10-year goal) }\end{array}$ \\
\hline
\end{tabular}

* Sensitivity at high latitude after a 2-year survey for a 5- $\sigma$ detection, units $10^{-8} \mathrm{~cm}^{-2} \mathrm{~s}^{-1}$, for $E>100 \mathrm{MeV}$.

"Diameter of $95 \%$ confidence region; range: bright sources to sources of flux $10^{-8} \mathrm{~cm}^{-2} \mathrm{~s}^{-1}(E>100 \mathrm{MeV})$.

\section{ADVANCES WITH GLAST}

The only external galaxy that EGRET detected in the light of its interstellar gamma-ray emission was the Large Magellanic Cloud (LMC), which was not spatially resolved (10). GLAST will be able to map the diffuse gamma-ray emision of the LMC, as well as the fainter Small Magellanic Cloud (SMC) and Andromeda (M31) galaxies. 

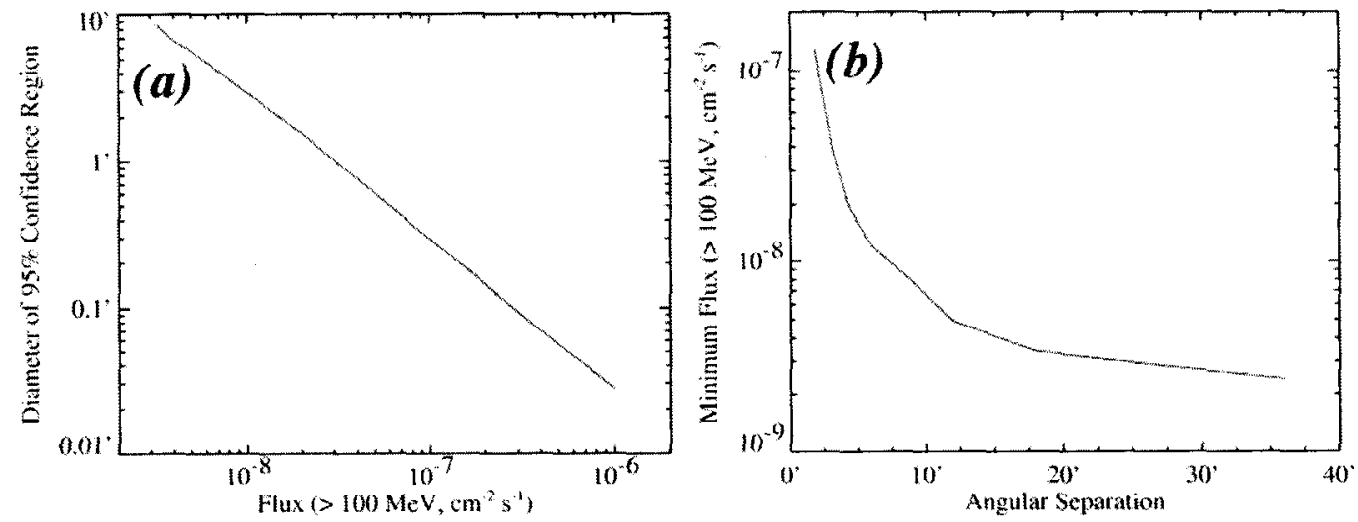

FIGURE 1. Expected performance of GLAST for localizing and resolving point sources. (a) Source localization at high latitudes. The position uncertainties for the brightest sources likely will be limited to $10-20^{\prime \prime}$ by uncertainty in spacecraft pointing and instrument alignment. (b) Minimum flux required to resolve two closely-spaced sources of equal flux. For both figures, the sources are assumed to have $E^{-2}$ photon spectra and to be observed at high latitudes in a one-year sky survey.

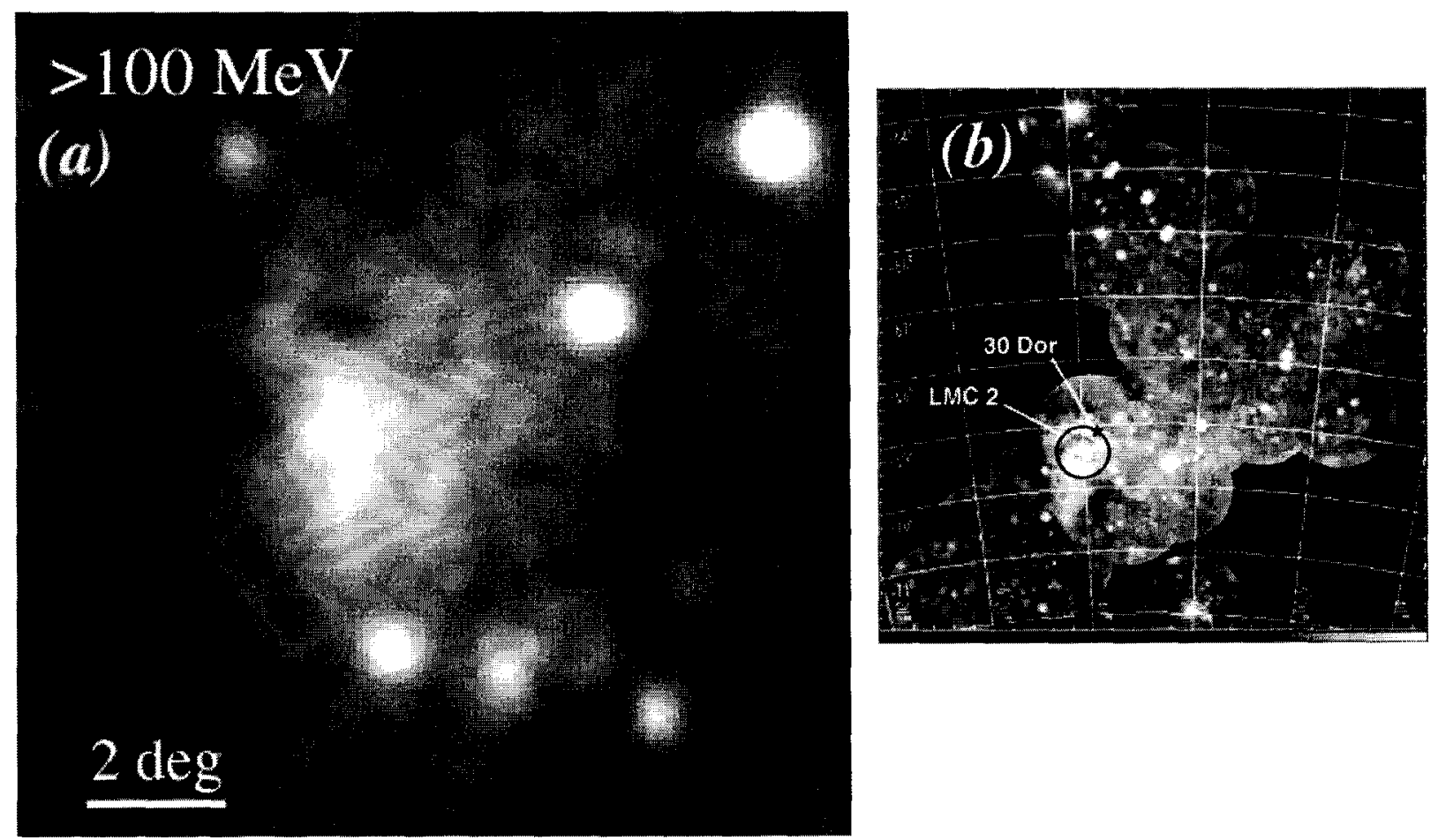

FIGURE 2. (a) Simulated map of the LMC in gamma rays (> $100 \mathrm{MeV}$ ) from a two-year sky survey with GLAST. The simulation is based on a model of the LMC by Sreekumar (12) and also includes foreground diffuse emission from the Milky Way and an isotropic background consisting of a distribution of faint point sources. (b) The LMC in 3/4-keV X-rays, from a mosaic of pointed observations with ROSAT (8). The intense emission regions of 30 Doradus and LMC superbubble 2 are indicated.

\section{$L M C$}

The LMC will be well-resolved by GLAST (Fig. 2). The cosmic-ray distribution can be studied in detail by analyzing the gamma-ray data together with $21-\mathrm{cm} \mathrm{H} \mathrm{I}$ and $2.6-\mathrm{mm} \mathrm{CO}$ surveys of the interstellar medium of the galaxy (see, e.g., (2)). GLAST data should reveal the degree of enhancement of cosmic-ray density in the vicinity of the massive star-forming region 30 Dor and the associated superbubble LMC 2 (6); see the X-ray image in Fig. 2. The LMC 2 superbubble is among the largest of several prominent superbubbles in the LMC, 


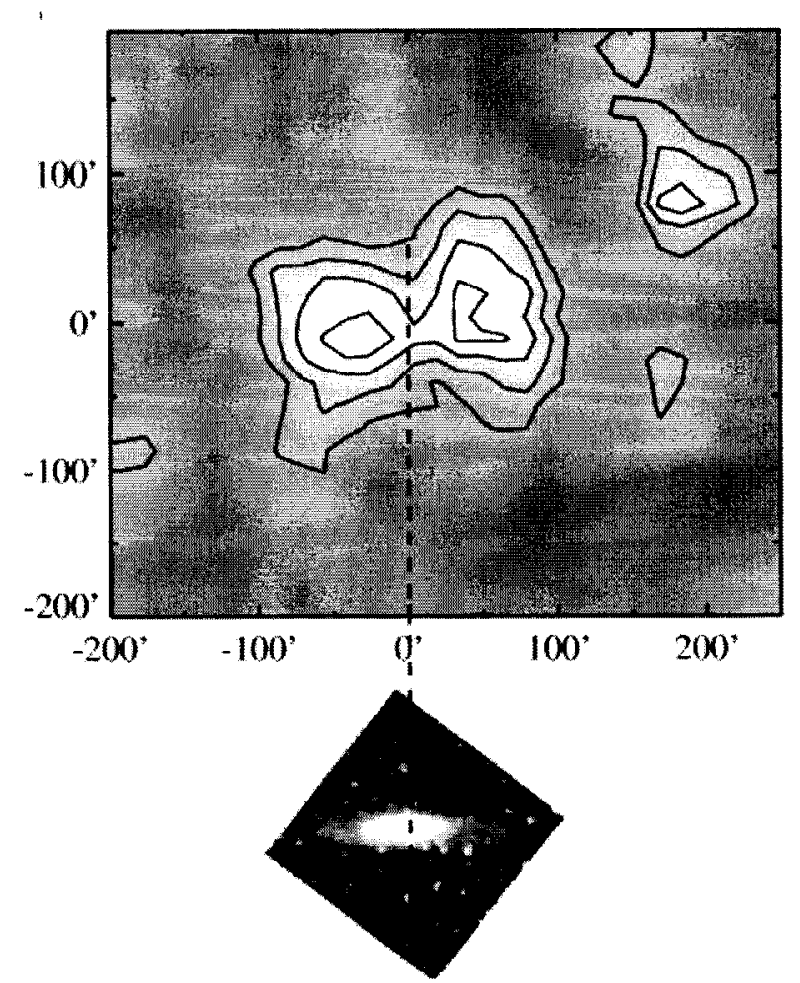

FIGURE 3. Simulated map of M31 from a five-year sky survey with GLAST (approximately equivalent to 6 months of observations with $\mathrm{M} 31$ within $30^{\circ}$ of the center of GLAST's field of view). The image shows gamma-rays with energies $>1 \mathrm{GeV}$, and has been smoothed to reduce statistical fluctuations. The simulated point source in the upper right indicates the angular resolution of the image, and the inset shows the location and extent of the optical disk of the galaxy. The diffuse emission was modelled based on the distribution of gas in M31, which extends much further than the optical disk, and the EGRET upper limit for the galaxy (1). Contours are spaced by $2 \times 10^{-7}$ $\mathrm{cm}^{-2} \mathrm{~s}^{-1} \mathrm{sr}^{-1}$ from $2.2 \times 10^{-6} \mathrm{~cm}^{-2} \mathrm{~s}^{-2} \mathrm{sr}^{-1}$.

most of which represent regions like 30 Dor that are further evolved. If superbubbles are sources of cosmic rays distinct from individual supernovas (e.g., (4)), superbubble 2 , which subtends $1^{\circ}$, may be marginally spatially resolved by GLAST. The extent to which the diffuse emission of the LMC can be attributed to unresolved gammaray pulsars has been considered by Hartmann et al. (3) and Zhang \& Cheng (14). The expectation is that the pulsar contribution could be significant, up to $35 \%$, for energies $>1 \mathrm{GeV}$. GLAST is unlikely to detect individual pulsars in the LMC, but may be able to address the question of the pulsar fraction with a sensitive measurement of the high-energy spectrum.

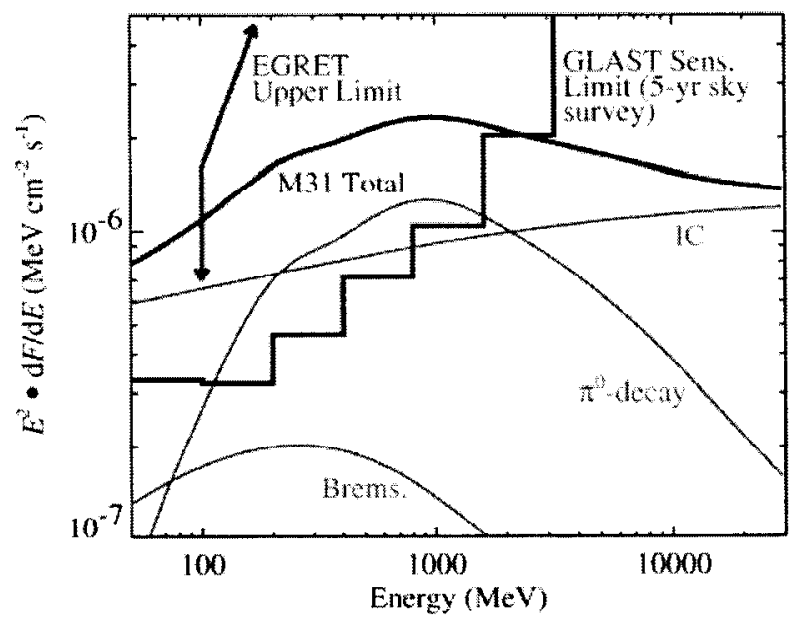

FIGURE 4. Simulated spectrum of M31, obtained by scaling the luminosity spectrum of the whole Milky Way derived by Strong et al. (13) (4-kpc halo model) to match the upper limit flux of Blom et al. (1) for M31. The differential flux sensitivity of GLAST for a five-year sky survey and the EGRET upper limit of Blom et al. are also shown, along with the individual components of the overall spectrum: inverse Compton, Bremsstrahlung, and $\pi^{0}$-decay.

\section{$S M C$}

Detection of the diffuse gamma-ray flux of the SMC by GLAST will be useful to verify conclusions about the galactic origin of cosmic rays (e.g., Sreekumar et al. (11) based on EGRET data). The non-detection of the SMC, with a 2- $\sigma$ upper limit of $5 \times 10^{-8} \mathrm{~cm}^{-2} \mathrm{~s}^{-1}(>100 \mathrm{MeV})$ led Sreekumar et al. to conclude that the most likely model for the distribution of cosmic rays in the SMC is one for which the galaxy is disintegrating and cosmic rays are only very poorly confined. In this circumstance, the predicted flux is $(2 \pm 3) \times 10^{-8} \mathrm{~cm}^{-2} \mathrm{~s}^{-1}(>100 \mathrm{MeV})$ (9), well within the reach of GLAST.

\section{M31}

The EGRET 2- $\sigma$ upper limit for the gamma-ray flux of M31 is $1.6 \times 10^{-8} \mathrm{~cm}^{-2} \mathrm{~s}^{-1}(>100 \mathrm{MeV})$, which is much less than the flux of the Milky Way at M31 (1). The cosmic-ray densities in M31 are certainly lower than in the Milky Way, and it has less ongoing massive star formation. At a flux level of $1 \times 10^{-8} \mathrm{~cm}^{-2} \mathrm{~s}^{-1}$ (>100 $\mathrm{MeV})$, GLAST will resolve the diffuse gamma-ray emission along the major axis of M31, to provide information about the relationship between cosmic rays, star formation rate, and interstellar gas on a large scale (Fig. 3). GLAST may also measure the distribution of cosmic rays 
in the halo of M31. Spectral measurements may allow a global assessment of inverse-Compton, electronBremsstrahlung, and $\pi^{0}$ decay contributions to the interstellar emission (Fig. 4). The unexplained "GeV excess" for the Milky Way (5) will also be detected if present in M31. From the gamma-ray spectra of the Milky Way and M31 the contribution of normal galaxics to the extragalactic gamma-ray background can begin to be assessed.

Detailed studies of M31 that will be possible with GLAST will benefit from further development of cosmicray models for the Milky Way, for which results from gamma-ray observations can be checked with direct observations of cosmic rays.

\section{CONCLUSIONS}

For the first time, GLAST will enable spatial and spectral studies of diffuse gamma rays from external galaxies. Diffuse, high-energy gamma rays are diagnostic of cosmic-ray densities, which especially for the proton component are difficult to determine from observations at other wavelengths. When considered together with Xray and radio observations, GLAST data promise a fairly complete understanding of the production, propagation, and confinement of cosmic rays in Local Group galaxies.

IVM acknowledges support from an NAS/NRC Senior Associateship.

\section{REFERENCES}

1. Blom, J. J., et al., Astrophys. J. 516, 44, (1999).

2. Cohen, R. S., et al., Astrophys. J. 331, L95, (1988).

3. Hartmann, D. H., Brown, L. E., and Schnepf, N., Astrophys. J. 408, L13, (1993).

4. Higdon, J. C., et al., Astrophys. J., 509, 33, (1998).

5. Hunter, S. D., et al., Astrophys. J. 481, 205, (1997).

6. Meaburn, J., MNRAS 192, 365, (1980).

7. Ormes, J. F., et al., these proceedings, (2000), astro-ph/0003270.

8. Snowden, S. L., and Petre, R., Astrophys. J. 436, L123, (1994).

9. Sreekumar, P., and Fichtel, C. E., Astron. and Astrophys. 251, 447, (1991).

10. Sreekumar, P., et al., Astrophys. J. 400, L67, (1992).

11. Sreekumar, P., et al., Phys. Rev. Lett. 70, 127, (1993).

12. Sreekumar, P., priv. comm., 1999.

13. Strong, A. W., Moskalenko, I., and Reimer, O., Astrophys. $J ., 537$, in press (2000), astro-ph/ 9811296 .

14. Zhang, L., and Cheng, K. S., MNRAS, 294, 729, (1998). 